\title{
RIN and Transmission Performance Improvement Using Second Order and Broadband First Order Forward Raman Pumping
}

\author{
Md Asif Iqbal, Mingming Tan, Atalla El-Taher and Paul Harper \\ Aston Institute of Photonic Technologies (AIPT), Aston University, Birmingham, UK \\ iqbalm7@aston.ac.uk
}

\begin{abstract}
Low RIN bidirectionally pumped Raman amplification is studied. We investigate the importance of the $1^{\text {st }}$ order forward pump generation technique and optimize the forward pump characteristics to achieve RIN comparable with backward only pumping and increased transmission reach.
\end{abstract}

Keywords-Relative intensity noise; coherent communication; Raman amplification

\section{INTRODUCTION}

In optical fibre communications systems distributed Raman amplification is used to improve the signal to noise ratio compared with discrete amplification. Bidirectional dual order pumping further improves the optical to signal ratio (OSNR) by reducing the signal power variation along the span compared with conventional first order bidirectional and backward pumping [1,2]. However the improvement in OSNR is counteracted by the impact of relative intensity noise (RIN) transfer from forward pump(s) to signal. Recently many methods have been applied to reduce this RIN transfer such as reducing the front end reflections [3,4], using incoherent pumping [5].

In this paper we use a technique which has some similarity with [5] in that the spectral properties of the forward pump are optimised to improve RIN and consequently transmission performance. We use bidirectionally pumped Raman amplification which has dual order forward Raman pumping: a $1365 \mathrm{~nm}$ Raman fibre laser provides the $2^{\text {nd }}$ order pump and the first order pump is broadband centred at $1455 \mathrm{~nm}$. This technique allows signal RIN suppression to a level comparable to backward only pumping but we show that the method used to generate the broadband first order pump has a significant impact on both RIN and transmission performance.

We compare two methods to generate the $1^{\text {st }}$ order forward pump experimentally. With optimisation the transmission performance of a $10 \times 100 \mathrm{G}$ DP-QPSK WDM system was improved compared with backward only pumping but performance was degraded if the wrong pump generation technique was used. We achieve a signal RIN level of $\sim-140 \mathrm{~dB} / \mathrm{Hz}$ with bidirectional pumping and a transmission distance of $\sim 7500 \mathrm{~km}$ which is an increase of $\sim 400 \mathrm{~km}$ compared with conventional backward pumping.

\section{Distributed RAman AmPlifiER SPAN SETUP}

Fig. 1 shows the configuration used for the Raman amplifier. In the transmission fibre span (Fig. 1(a)), backward dual order pumping is formed by a $2^{\text {nd }}$ order pump $(1365 \mathrm{~nm})$ and a high reflectivity ( $\sim 95 \%)$ fibre Bragg grating (FBG) centred at $1455 \mathrm{~nm}$. The inclusion of the narrowband $(\sim 1 \mathrm{~nm})$ FBG gives random lasing at $1455 \mathrm{~nm}$ near the output end of the span $[2,3]$. Forward pumping consisted of a $2^{\text {nd }}$ order $1365 \mathrm{~nm}$ pump and a broadband $1^{\text {st }}$ order pump with $3 \mathrm{~dB}$ bandwidth $18 \mathrm{~nm}$ and centred at $1455 \mathrm{~nm}$. Only a low $1^{\text {st }}$ order power is required to provide a seed for the Raman amplification and this first order pump seed was generated by backward pumping $10 \mathrm{~km}$ of G652 standard single mode fibre (SSMF) at $1365 \mathrm{~nm}$. The $10 \mathrm{~km}$ fibre length was used as a compromise between the pump power requirement and $1^{\text {st }}$ order power generation efficiency. The two different methods used to generate this are shown in Fig. 1 (b) and (c).

Scheme-1 shown in Fig. 1 (b) used an open-cavity random fibre laser based configuration without any reflections from either end of the seed generation fibre whereas scheme-2 in

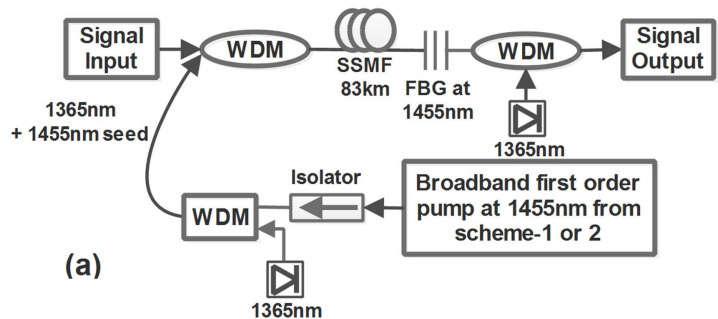

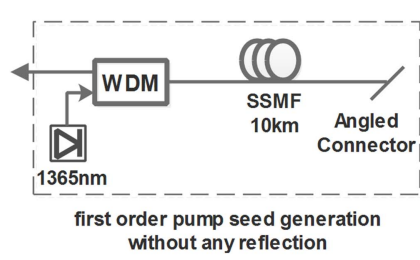

(b)

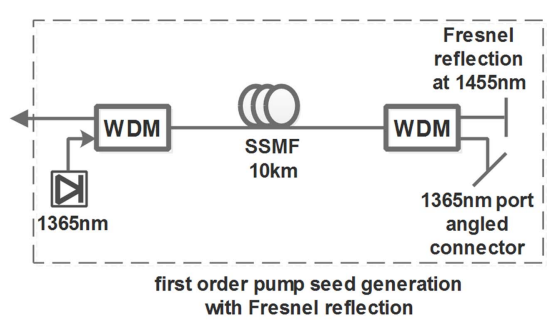

(c)

Scheme-2

Fig. 1: (a) Schematic of broadband $1^{\text {st }}$ order forward pump based dual order bidirectional Raman span (b) scheme-1: $1^{\text {st }}$ order pump seed generation without any cavity reflections (c) scheme-2: $1^{\text {st }}$ order pump seed generation with $3.4 \%$ Fresnel back reflections 

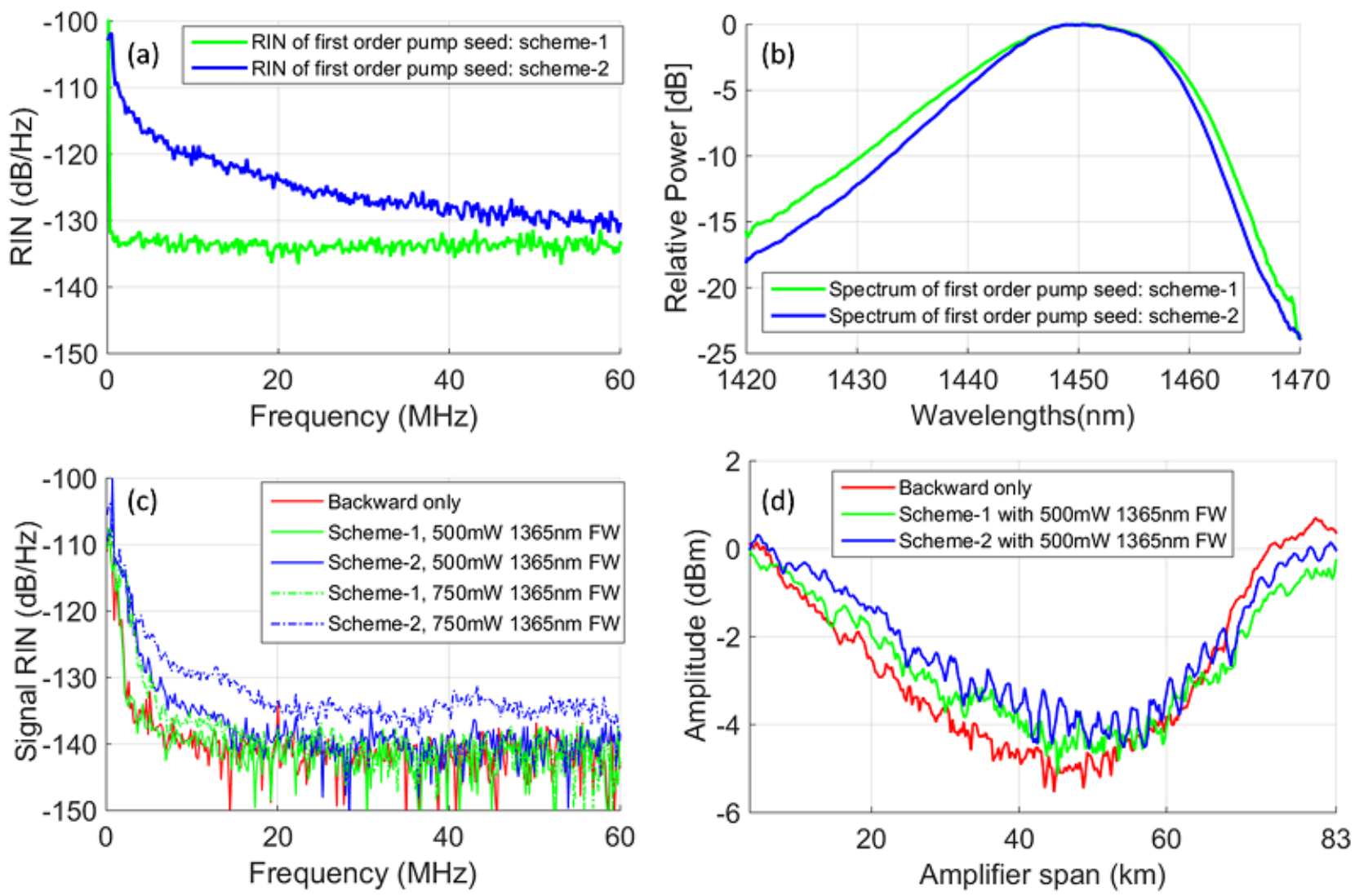

Fig. 2: (a) RIN of $1^{\text {st }}$ order broadband pump seed from two different schemes (b) spectra of $1^{\text {st }}$ order broadband pump seed generated from scheme-1 and 2 (c) signal RIN at $194 \mathrm{THz}$ using different Raman pumping schemes (d) signal power profile at 194THz using different Raman pumping schemes

Fig. 1(c) used a very low ( $3.4 \%)$, broadband (1420 1480nm) Fresnel reflection from the far end of the fibre.

Scheme-1 generated $3 \mathrm{~mW}$ of first order pump power using $4.5 \mathrm{~W}$ at $1365 \mathrm{~nm}$. In scheme- 2 the $1365 \mathrm{~nm}$ pump power that could be used was limited to $3.5 \mathrm{~W}$ : higher pump power gave lasing at the Stokes wavelength due to feedback from double Rayleigh backscattering (DRB) [7]. Scheme-2 gave a $1 \mathrm{~mW} 1^{\text {st }}$ order seed at this maximum $1365 \mathrm{~nm}$ pump power.

The $1^{\text {st }}$ order broadband seed was combined with the $1365 \mathrm{~nm}$ pump through an isolator. The isolator blocked $1455 \mathrm{~nm}$ scattered light from the transmission span from entering the seed generation set-up.

\section{RIN CHARACTERISATION}

The signal RIN performance mainly depends on the RIN transfer from the forward $1^{\text {st }}$ and $2^{\text {nd }}$ order pumps in dual order bidirectional amplifiers.

Fig. 2(a) shows the comparison of the RIN of the $1^{\text {st }}$ order pump generated using scheme-1 and 2. It can be seen that for scheme-1 the RIN level is very low and remains constant at $\sim-135 \mathrm{~dB} / \mathrm{Hz}$, whereas in scheme-2 the RIN is higher, more than $-120 \mathrm{~dB} / \mathrm{HZ}$ in the low frequency range. Spectra of the forward $1^{\text {st }}$ order seeds shown in Fig. 2(b) have a similar $3 \mathrm{~dB}$ bandwidth of $\sim 18 \mathrm{~nm}$.
To Raman pump the transmission fibre span forward $1365 \mathrm{~nm}$ pumping was used with two different powers, $500 \mathrm{~mW}$ and $750 \mathrm{~mW}$. The backward $1365 \mathrm{~nm}$ pump power was varied between 1 and $1.2 \mathrm{~W}$ to maintain a $16.5 \mathrm{~dB}$ on-off gain compensating the loss of the $83.32 \mathrm{~km}$ standard fibre span.

Fig. 2(c) shows the signal RIN performance and a comparison with backward pumping only. Scheme-1 shows the best performance and is comparable with backward only pumping. The slight increase in signal RIN level in shceme-1 with $750 \mathrm{~mW}$ of $1365 \mathrm{~nm}$ pump was mainly due to the transfer from the higher order pump but still remains lower than that of scheme-2 with $500 \mathrm{~mW}$. Fig. 2(d) shows the improved signal power profiles of both the schemes compared with backward pumping only.

\section{TRANSMISSION RESULTS AND DISCUSSION}

The long haul coherent transmission performance was compared. The lowest signal RIN configurations in both the schemes with $500 \mathrm{~mW}$ forward $1365 \mathrm{~nm}$ pump power were used for performance comparison with the backward pumping only case. A re-circulating loop setup with $10 \times 100 \mathrm{G}$ DP-QPSK WDM transmission setup was used as shown in Fig. 3. The transmitter consisted of $10 \mathrm{WDM}$ channels $(100 \mathrm{GHz}$ spaced from $194.3 \sim 193.4 \mathrm{THz}$ ) combined with a $100 \mathrm{kHz}$ laser as "channel under test", a polarisation maintaining EDFA to amplify 30GBaud DP-QPSK modulated WDM signals and a 


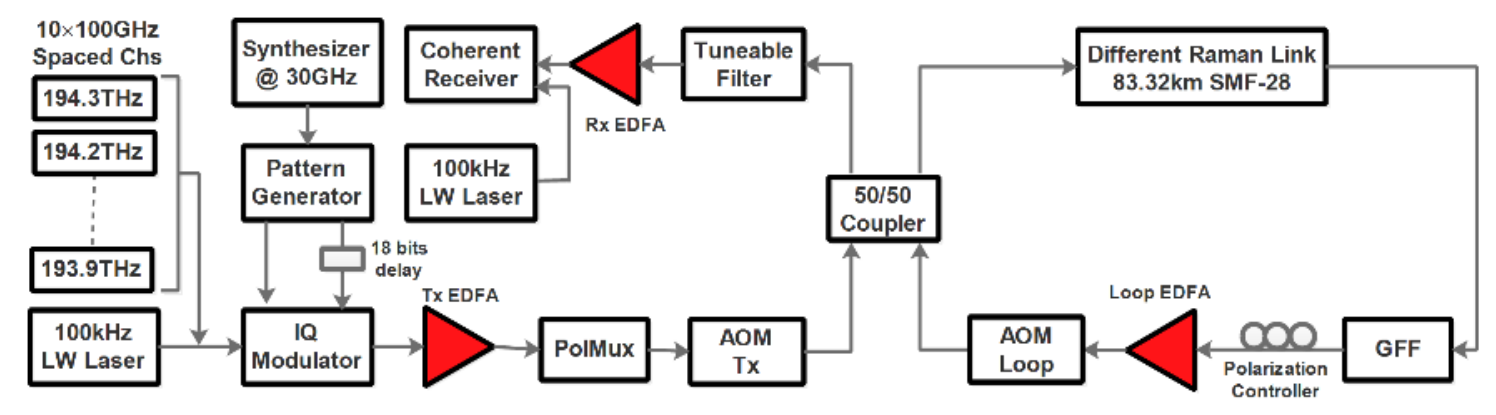

Fig. 3: Experimental setup for long-haul coherent transmission
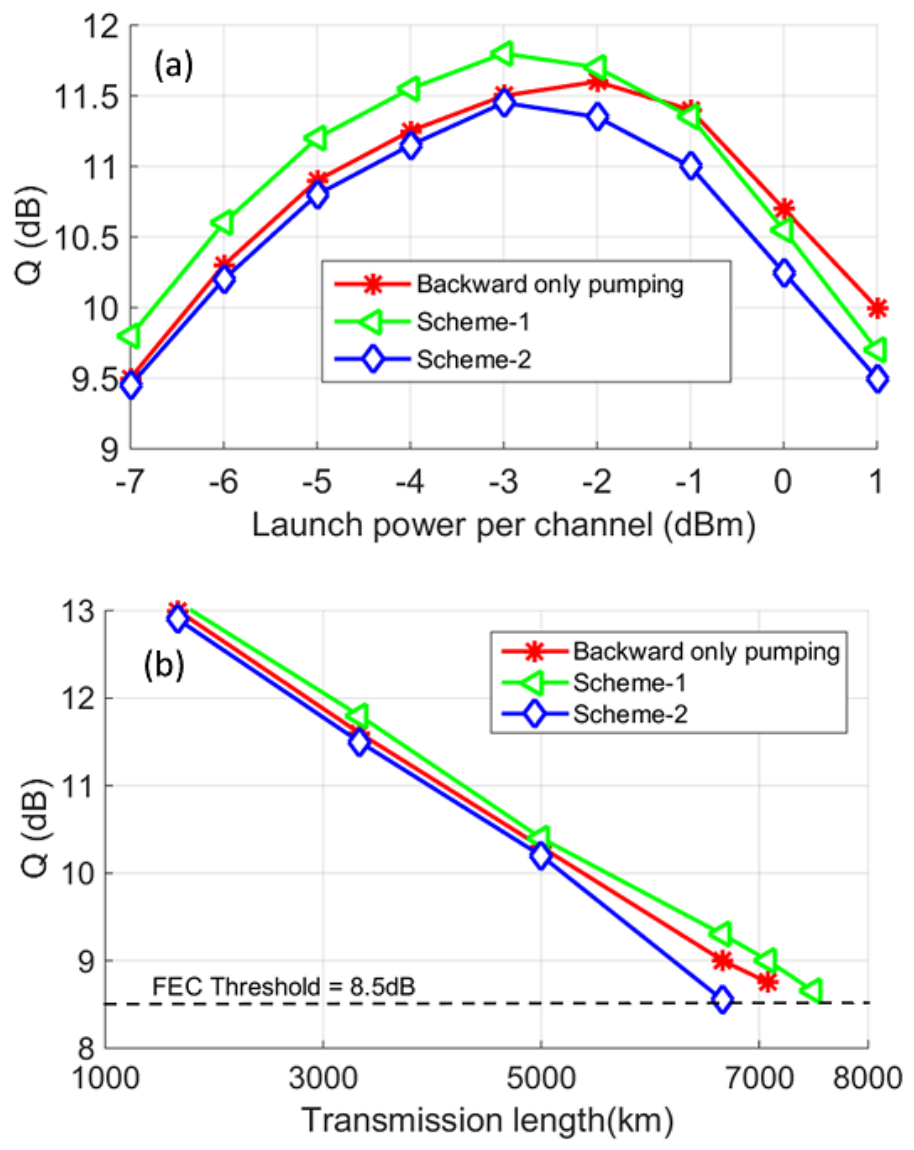

Fig. 4: (a) Q factors versus launch power per channel sweep for the middle WDM channel at $194 \mathrm{THz}$ (b) Q factors versus transmission distances at $194 \mathrm{THz}$

polarisation multiplexer. The total loss of the $83.32 \mathrm{~km}$ Raman span was $\sim 17.6 \mathrm{~dB}$ including $16.5 \mathrm{~dB}$ of fibre loss and $\sim 1.1 \mathrm{~dB}$ from pump/signal combiner pairs. The additional insertion loss of $\sim 12 \mathrm{~dB}$ from gain flattening filter (GFF), 50/50 coupler and acousto-optic modulator (AOM) was compensated by using a dual-stage EDFA at the end of the loop. Finally, the output signal was de-multiplexed and amplified by a tuneable filter and an EDFA respectively at the receiver. A polarisation diverse coherent receiver with $80 \mathrm{GSa} / \mathrm{s}, 36 \mathrm{GHz}$ bandwidth oscilloscope was used to detect the signal and then offline digital signal processing (DSP) was applied for post processing. Actual bit error counting over two million bits was used for $\mathrm{Q}$ factors calculation.

Fig. 4(a) shows the $Q$ factors versus signal (194THz) launch power per channel at $3333 \mathrm{~km}$ for different Raman pumping schemes. Q factors improvement of $0.2 \mathrm{~dB}$ and $0.4 \mathrm{~dB}$ compared with backward pumping only and scheme-2 respectively were observed with scheme-1 at optimum launch power of $-3 \mathrm{dBm}$ per channel. This additional improvement comes from comparable signal RIN performance as backward pumping only and improved noise performance by using forward pumping. Although scheme-2 has similar signal power profile to scheme-1 (Fig. 2(d)) and the same 1365nm forward pump power, the worst performance with $0.2 \mathrm{~dB}$ degradation of Q factor compared with backward pumping only was mainly due to the high signal RIN transferred from high RIN of the $1^{\text {st }}$ order pump seed. The $1 \mathrm{~dB}$ decrease in optimum launch power in both the schemes compared to backward only pumping was expected and caused by higher average signal power along the span leading to additional nonlinearity degradation. Fig. 4(b) shows the Q factors versus transmission distance with different Raman configurations at optimum launch power as shown in Fig. 4(a). Scheme-1 shows the maximum transmission distance of $7499 \mathrm{~km}$ with an additional $\sim 400 \mathrm{~km}$ and $\sim 800 \mathrm{~km}$ reach enhancement relative to backward only pumping and scheme-2 respectively, due to improved RIN and OSNR performance.

\section{CONCLUSIONS}

We have demonstrated that RIN transfer in a bidirectionally pumped distributed Raman amplifier can be reduced by using a broadband $1^{\text {st }}$ order Raman pump. We show that the generation process for this broadband pump has an impact on RIN and consequently transmission performance. With optimisation the performance of the forward pumped system had RIN comparable with backward only pumping but improved transmission performance. However with an inappropriate broadband pump generation scheme both RIN and transmission performance were degraded.

In conclusion, for a $10 \times 100 \mathrm{G}$ DP-QPSK WDM system, broadband $1^{\text {st }}$ order pump generation using an open-cavity random lasing configuration allowed a $\sim 400 \mathrm{~km}$ transmission reach increase to $\sim 7500 \mathrm{~km}$ compared with conventional backward pumping only. 


\section{ACKNOWLEDGMENT}

This work was funded by EU FP7 ITN programme ICONE (No. 608099) and UK EPSRC Programme Grant UNLOC $\mathrm{EP} / \mathrm{J} 017582 / 1$.

\section{REFERENCES}

[1] J. Ania-Castañón, "Quasi-lossless transmission using second-order Raman amplification and fibre Bragg gratings," Opt. Express, vol. 12, no. 19 , pp. 4372-4377, 2004.

[2] M. Tan, P. Rosa, S. T. Le, I. D. Philips, and P. Harper, "Evaluation of 100G DP-QPSK long-haul transmission performance using second order co-pumped Raman laser based amplification," Opt. Express, vol. 23, pp. 22181-22189, 2015.
[3] M. Tan, P. Rosa, S. T. Le, Md. A. Iqbal, I. D. Phillips, and P. Harper, "Transmission performance improvement using random DFB laser based Raman amplification and bidirectional second-order pumping," Opt. Express, vol. 24, pp. 2215-2221, 2016.

[4] G. Rizzelli et al., "Impact of input FBG reflectivity and forward pump power on RIN transfer in ultralong Raman laser amplifiers," Opt. Express, vol. 24, pp. 29170-29175, 2016.

[5] M. Morimoto; H. Ogoshi; J. Yoshida; S. Takasaka; A. Sano; Y. Miyamoto, "Co-propagating dual-order distributed Raman amplifier utilizing incoherent pumping," IEEE Photonics Technol. Lett., vol. PP, no.99, pp.1-1, 2017.

[6] S. K. Turitsyn, S. A. Babin, A. E. El-Taher, et al., "Random distributed feedback fibre laser," Nature Photon, 4(4), pp. 231-235, 2010.

[7] D. V. Churkin, S. A. Babin, A. E. El-Taher, et al., "Raman Fiber Lasers with a Random Distributed Feedback Based on Rayleigh Scattering," Phys. Rev. A, 82(3), pp. 033828, 2010. 